\section{Folatos y riesgo de cáncer de mama: revisión sistemática}

\author{
CECILIA CASTILLO-L. ${ }^{1}$, JOSEP A. TUR ${ }^{1}$, RICARDO UAUY ${ }^{2}$
}

\section{Folate and breast cancer risk. A systematic review}

An increased folate intake may be beneficial in deficient populations. However, in women with adequate levels it may not deliver additional benefits while it may increase the risk for some forms of cancer. A systematic literature review of benefits or risks of folate in the development of breast cancer was performed using MEDLINE, systematic review of selected articles and references of the selected articles looking specifically at serum folate levels, dietary folate intake or total folate intake and the risk of developing breast cancer. Fourteen case-control studies, fourteen cohort studies, seven case-control nested studies, two randomized trials and two meta-analyses were selected for analysis based on pre-established criteria. The reviewed evidence does not support the hypothesis that higher intakes of dietary folate reduce the risk for breast cancer. Some studies showed a higher risk of breast cancer in populations exposed to high folate intake post fortification, especially when folic acid is used. The results support the need to be cautious and to limit the exposure of women to high intakes of folic acid, especially in countries with mandatory food fortification.

(Rev Med Chile 2012; 140: 251-260).

Key words: Breast neoplasms; Folic acid; Review.

$\mathrm{E}$ 1 cáncer de mama es el más frecuente entre las mujeres, estimándose una incidencia anual a nivel mundial cercana a un millón de nuevos casos ${ }^{1}$. Su etiología no es completamente entendida ya que su desarrollo está relacionado con múltiples factores: genéticos, edad, raza, eventos reproductivos y uso exógeno de hormonas entre otros ${ }^{2}$. En estudios de cáncer de mama se ha descrito el hallazgo de tejidos mamarios con ADN hipometilado y con un mayor contenido de folatos ${ }^{3}$. Se postula que su efecto sobre el cáncer de mama podría estar mediado por los niveles de ácido fólico, que actuaría modificando la regulación de la expresión de algunos genes, resultando en un silenciamiento de algunos o bien promoviendo el crecimiento de tumores que presentan una mayor expresión de receptores de folatos ${ }^{4,5}$.

Considerando que en Chile la implementación de la fortificación obligatoria de la harina de trigo ha determinado un importante aumento de los niveles de folato sérico ${ }^{6-8}$, hemos desarrollado la presente revisión con el objetivo de identificar los beneficios o riesgos descritos en la literatura científica en diferentes modelos de estudios en relación a folatos y riesgo para desarrollar de cáncer de mama.

\section{Material y Método}

Para responder al objetivo de esta revisión sistemática se efectuó una búsqueda en revistas científicas en inglés y español provenientes de la base de datos Medline, vía Pubmed. Se utilizaron los siguientes descriptores de ciencias de la salud, "Medical Subject Headings" (MESH): ácido fólico y neoplasma de mama. Como segunda búsqueda específica se incluyó la palabra folato. Se encontraron 953 artículos de los cuales se seleccionaron los que describían y analizaban la relación entre nivel de folatos séricos y/o ingesta de folatos (dietarios, totales consumidos) y el riesgo para desarrollar cáncer de mama en humanos (Figura 1). Dos revisores independientes, considerando los criterios de selección establecidos, revisaron y excluyeron 
los artículos que no alcancaban el umbral mínimo de calidad para su diseño según la versión española "Critical Appraisal Skills Programme" (CASPe) ${ }^{9}$ y aquellos en que no se podía garantizar el control de los sesgos. Las referencias asociadas en los artículos seleccionados fueron revisadas en forma manual para seleccionar otras publicaciones relevantes en relación a los criterios de selección establecidos. Los criterios de inclusión exclusión y los estudios seleccionados se muestran en las Tablas 1 y 2 .

\section{Resultados}

\section{Estudios de caso control}

Los resultados de los estudios varían desde la inexistencia de una relación ${ }^{10,11}$ hasta un aumento del riesgo de cáncer de mama ${ }^{12}$ o bien muestran asociaciones no significativas ${ }^{13,14}$. En algunos se observa un efecto significativamente protector con mayores consumos de folatos, especialmente en la menopausia ${ }^{15-18}$. En otros el riesgo es mayor en la premenopausia ${ }^{12}$.

$\mathrm{Al}$ analizar por tipo de folatos consumidos, algunos autores describen un menor riesgo de cáncer de mama en relación a ingesta elevada de folatos dietarios en mujeres que no consumían suplementos de ácido fólico ${ }^{10}$, sugiriendo que los folatos naturales podrían tener un efecto protector.

Otras publicaciones describen un mayor riesgo de cáncer de mama asociado a niveles elevados de folato totales y plasmáticos, especialmente en mujeres premenopáusicas y portadoras de algunos polimorfismos del gen de la enzima 5-10 metilentetrahidro-folato-reductasa (MTHFR C677T y A1298C $)^{14}$. Otros muestran una disminución del riesgo de cáncer de mama independiente del nivel de folatos dietarios, especialmente en las variantes homocigotos de la MTHFR C677T y C1298A (TT y CC $)^{13}$. Este menor riesgo se observaría especialmente en la postmenopausia sólo cuando el nivel de folatos séricos es elevado, especialmente en el genotipo TT de la MTHFR C677 $\mathrm{T}^{19}$ o con una mayor ingesta de folatos dietarios ${ }^{10,15,20,21}$.

La inclusión en el análisis de diferentes subtipos de receptores hormonales de la mama sugiere que el riesgo de cáncer de mama varía dependiendo del grado de metilación ${ }^{22}$, describiéndose un mayor riesgo con bajas ingestas de folatos y de metionina cuando las islas $\mathrm{CpG}$ de los receptores estrogénicos (ER) se encuentran metilados y un menor riesgo, aunque no significativo, en cánceres no metilados.

El consumo de alcohol es otro factor de riesgo asociado a un mayor riesgo de cáncer de mama, especialmente cuando la ingesta de folatos es baja debido al mayor requerimiento observado ${ }^{23}$, sin embargo, esta asociación no queda claramente demostrada ${ }^{16,22}$.

El contenido de vegetales crudos y granos integrales en la dieta, así como el folato dietario

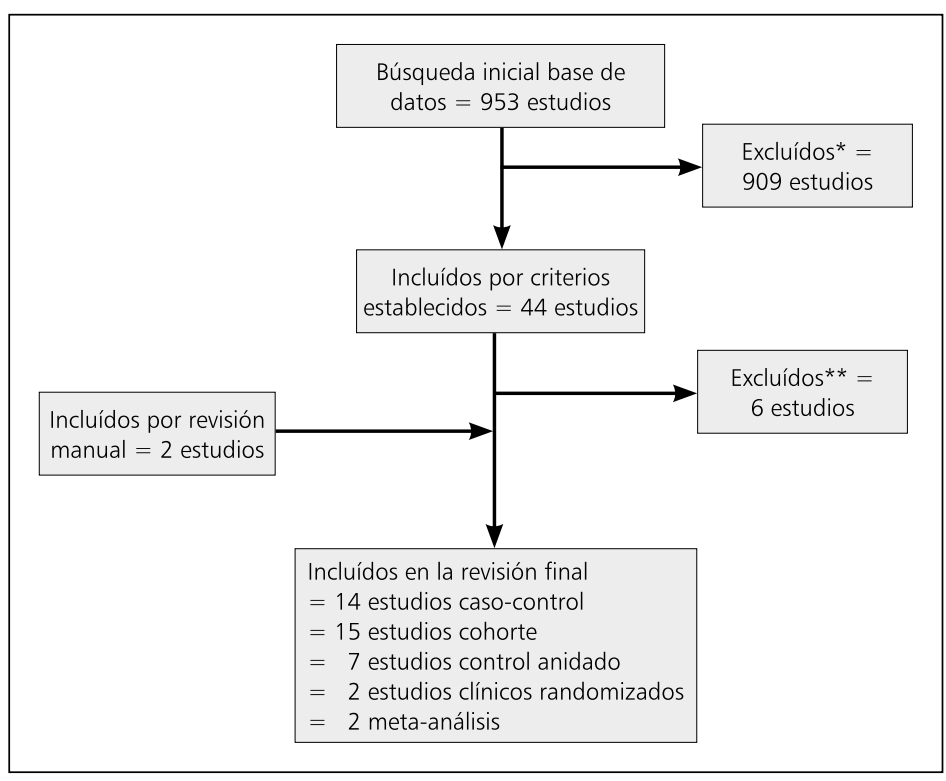

Figura 1. Etapas de la selección de artículos para Revisión Sistemática. ${ }^{*} /{ }^{* *}$ Criterios en Tabla 1. 


\section{Tabla 1. Criterios de Inclusión y Exclusión}

\section{Criterios Inclusión}

Estudios que establecen riesgo (OR, RR) entre diferentes ingesta de folato (totales, dietarios, séricos, ácido fólico) y neoplasia de mama

Estudios que establecen riesgos (OR, RR) entre diferentes niveles de folato sérico y neoplasia de mama

Estudios que establecen riesgo (OR, RR) entre diferentes ingesta de folato (totales, dietarios, séricos, ácido fólico) junto a otras vitaminas y neoplasia de mama

\section{Criterios Exclusión}

Resúmenes, Reportes de Casos, Editoriales, Revisiones*

Estudios clínicos de drogas para tratamiento de neoplasia de Mama*

Asociaciones de otros nutrientes y neoplasia de Mama*

Asociaciones entre otros marcadores bioquímicos y neoplasia de Mama*

No se entregan datos en relación a riesgos y diferentes niveles de folatos**

Estudios de cohorte con un seguimiento $<5$ años**

*Primera etapa de selección de estudios. ${ }^{*}$ Segunda etapa de selección de estudios. asociados a otros componentes como vitamina $\mathrm{C}$ y betacarotenos, podrían tener también un significativo efecto protector ${ }^{24}$.

\section{Estudios caso-control anidado}

Como en el modelo anterior, los resultados de estudios de casos anidados en cohortes muestran resultados conflictivos para la relación folatos y cáncer de mama que van desde la inexistencia de una asociación ${ }^{25,26}$ a la descripción de una relación inversa cuando se considera el nivel de folato plasmático, como describen Zhang et $\mathrm{al}^{27}$ en población norteamericana.

A diferencia de este último ${ }^{27}$, Lin et $\mathrm{a}^{28}$ encuentran que altos niveles de folatos plasmáticos ( $>15,8 \mathrm{ng} / \mathrm{ml}$ ) en mujeres premenopáusicas norteamericanas se asocian positivamente con cáncer de mama ( $R R=1,99$; IC 95\% 1,01-3,93; P Interacción 0,05$)$, especialmente tras la fortificación de alimentos (folatos dietarios más fortificados $>582 \mu \mathrm{g} / \mathrm{d})(\mathrm{RR}=1,24$; IC95\% 0,88-1,76; P 0,03) (Figura 2).

Otros autores describen que altos niveles de folatos plasmáticos (>17 nmol/L) se asocian con un aumento de cáncer en mujeres post-menopaúsicas homocigotas o heterocigotas para el alelo $\mathrm{T}$

Tabla 2. Estudios incluidos en la revisión

\begin{tabular}{|c|c|c|c|c|c|}
\hline \multicolumn{6}{|c|}{ Estudios caso-control } \\
\hline Autor* (Ref.) & $\begin{array}{c}\text { Año } \\
\text { publicación }\end{array}$ & País & Casos/controles & Edad (años) & Folatos analizados \\
\hline Levi (16) & 2001 & Suiza & $289 / 442$ & $23-74$ & Folato dietario \\
\hline Shrubsole (17) & 2001 & China & $1.321 / 1.382$ & $25-64$ & Folato dietario \\
\hline Sharp (13) & 2002 & Escocia & $62 / 66$ & $50-69$ & Folato dietario \\
\hline Adzersen (24) & 2003 & Alemania & $310 / 353$ & $25-75$ & Folato dietario \\
\hline Zhu (22) & 2003 & EE.UU. & $304 / 305$ & $20-64$ & Folato dietario y A.F \\
\hline Beilby (19) & 2004 & Australia & $341 / 109$ & $30-84$ & Folato sérico \\
\hline Shrubsole (20) & 2004 & China & $1.144 / 1.236$ & $25-64$ & Folato dietario \\
\hline Chen (10) & 2005 & EEUU & $1.481 / 1.518$ & 58,8 (promedio) & Folato total y dietario \\
\hline Shrubsole (21) & 2006 & China & $1.144 / 1.236$ & $25-64$ & Folato dietario \\
\hline Lajous (18) & 2006 & México & $475 / 1.391$ & $18-87$ & Folato dietario \\
\hline Chou (14) & 2006 & Taiwán & $146 / 285$ & $20-80$ & Folato plasmático \\
\hline Susuki (15) & 2008 & Japón & $456 / 912$ & $20-79$ & Folato dietario \\
\hline Ma (12) & 2009 & Brasil & $458 / 458$ & $20-74$ & Folato dietario \\
\hline Ma (11) & 2009 & Japón & $388 / 388$ & $20-74$ & Folato dietario \\
\hline
\end{tabular}


Tabla 2. Estudios incluidos en la revisión (continuación)

\begin{tabular}{|c|c|c|c|c|c|}
\hline \multicolumn{6}{|c|}{ Estudios caso-control anidado } \\
\hline Autor* (Ref.) & $\begin{array}{c}\text { Año } \\
\text { publicación }\end{array}$ & País & Casos/controles & Edad (años) & Folatos analizados \\
\hline Rohan (25) & 2000 & Canadá & $1.336 / 5.382$ & $>40$ & Folato dietario \\
\hline Zhang (27) & 2003 & EE.UU. & $712 / 712$ & 57,2 (promedio) & Folato plasmático \\
\hline Tjønneland (26) & 2006 & Dinamarca & $388 / 388$ & $50-64$ & Folato total, dietarioy A.F. \\
\hline $\operatorname{Lin}(28)$ & 2008 & EE.UU. & $848 / 848$ & $\geq 45$ & Folato plasmático \\
\hline Ericson (29) & 2009 & Suecia & $313 / 626$ & $>55$ & Folato plasmático \\
\hline Ericson (30) & 2009 & Suecia & $544 / 1.088$ & $>55$ & Folato dietario \\
\hline Ericson (31) & 2010 & Suecia & $204 / 408$ & $>55$ & Folato plasmático \\
\hline
\end{tabular}

\begin{tabular}{|c|c|c|c|c|c|c|}
\hline \multicolumn{7}{|c|}{ Estudios de Cohorte } \\
\hline Autor* (Ref.) & $\begin{array}{c}\text { Año } \\
\text { publicación }\end{array}$ & País & $\begin{array}{l}\text { Población } \\
\text { estudiada }\end{array}$ & $\begin{array}{l}\text { Edad } \\
\text { (años) }\end{array}$ & $\begin{array}{l}\text { Seguimiento } \\
\text { (años) }\end{array}$ & Folatosanalizados \\
\hline Sellers (35) & 2001 & EE.UU. & 34.387 & $55-69$ & 12 & Folato dietario y $\mathrm{A} . \mathrm{F}^{\ddagger}$ \\
\hline Sellers(33) & 2002 & EE.UU. & 34.393 & $55-69$ & 12 & Folato dietario y A.F. ${ }^{\ddagger}$ \\
\hline Cho (36) & 2003 & EE.UU. & 90.655 & $26-46$ & 8 & Folato total y dietario \\
\hline Feigelson (37) & 2003 & EE.UU. & 66.561 & $40-87$ & 5 & Folato total y dietario \\
\hline Baglietto (47) & 2005 & Australia & 17.447 & $40-69$ & 13 & Folato dietario \\
\hline Rossi (44) & 2006 & Australia & 1024 & $\begin{array}{c}57 \\
\text { (promedio) }\end{array}$ & $\begin{array}{l}29 \text { (Mortalidad) }^{\circ} \\
23 \text { (Morbilidad) }^{\circ}\end{array}$ & $\begin{array}{l}\text { Folato sérico y } \\
\text { del glóbulo rojo }\end{array}$ \\
\hline Lajous (48) & 2006 & Francia & 62.739 & $\begin{array}{l}\text { Post- } \\
\text { menopausia }\end{array}$ & 9 & Folato dietario \\
\hline Stolzenberg (38) & 2006 & EE.UU. & 25.400 & $55-74$ & 4,9 & Folato dietario y A.F..$^{\ddagger}$ \\
\hline Ericsson (43) & 2007 & Suecia & 11.699 & $\geq 50$ & 9,5 & Folato total y dietario \\
\hline Kabat (39) & 2008 & Canadá & 49.654 & 40.59 & 16,4 & Folato dietario \\
\hline Duffy (40) & 2009 & EE.UU. & 88.530 & $50-79$ & 5,5 & Folato total \\
\hline Larsson (41) & 2008 & Suecia & 61.433 & $\begin{array}{c}53,3 \\
\text { (promedio) }\end{array}$ & 17,4 & Folato dietario \\
\hline Maruti (32) & 2009 & EE.UU. & 35.023 & $50-76$ & 5,5 & $\begin{array}{l}\text { Folato total, dietario } \\
\text { y A.F. }\end{array}$ \\
\hline Stevens (45) & 2010 & EE.UU. & 70.756 & $50-74$ & 13 & Folato total y dietario \\
\hline Rosswall (42) & 2010 & Dinamarca & 26.224 & $50-64$ & 10,6 & Folato dietario y A.F. ${ }^{\ddagger}$ \\
\hline
\end{tabular}

\begin{tabular}{|c|c|c|c|c|c|}
\hline \multicolumn{6}{|c|}{ Estudios Clínicos Randomizados } \\
\hline Autor* (Ref.) & $\begin{array}{c}\text { Año } \\
\text { publicación }\end{array}$ & País & $\begin{array}{l}\text { Población interve- } \\
\text { nida/placebo }\end{array}$ & $\begin{array}{l}\text { Seguimiento } \\
\text { (años) }\end{array}$ & Suplementación \\
\hline Charles (50) & 2004 & Escocia & 3.187 & 36 & A.F. ${ }^{\ddagger} 0,2 \mathrm{mg}$ y $5 \mathrm{mg}$ \\
\hline Zhang (49) & 2008 & EE.UU. & $2.721 / 2.721$ & 7,3 & A.F. ${ }^{\ddagger}, 5$; Vit. $B_{6} 50$;Vit $B_{12} 1 \mathrm{mg}$ \\
\hline
\end{tabular}

\begin{tabular}{|lccl|}
\hline $\begin{array}{l}\text { Metaanálisis } \\
\text { Autor* (Ref.) }\end{array}$ & Año publicación & Estudios incorporados & Efecto estimado \\
Lewis (51) & 2006 & $\begin{array}{c}13 \text { estudios de cohorte } \\
\text { 9 estudios caso-control }\end{array}$ & $\mathrm{RR}=0,99(0,98-1,01)$ \\
& & OR $=0,91(0,87-0,96)$
\end{tabular}

*Sólo se indica el primer autor Ref.= Referencia. Folato total= Folato dietario+ ácido fólico contenido en suplementos vitamínicos. ${ }^{\ddagger}$ A.F. $=$ ácido fólico como suplemento vitamínico. ${ }^{\dagger}$ Folato total. ${ }^{+\dagger}$ Folato dietario. 


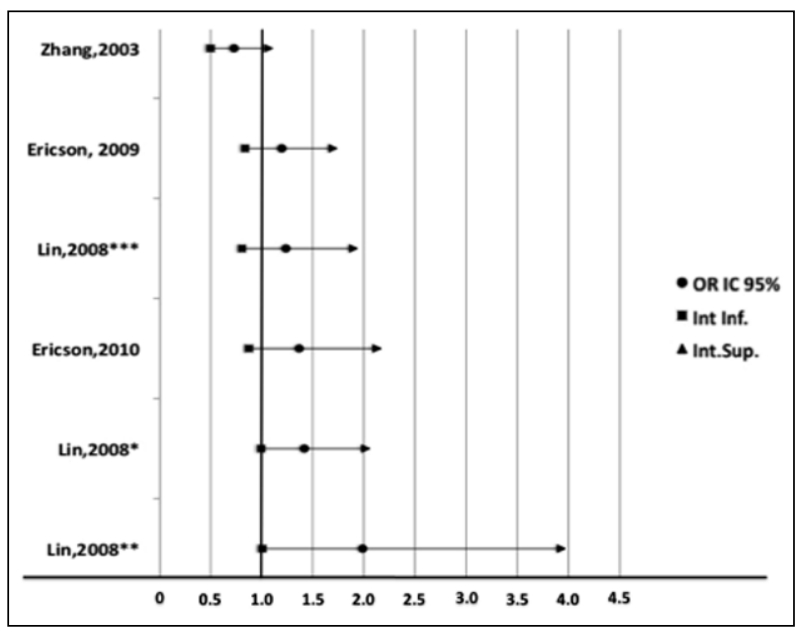

Figura 2. Folatos plasmáticos y riesgo de cáncer de mama. Estudios caso-control anidados. *Pre y Postmenopausia. **Pre-menoapausia. ***Postmenopausia. del polimorfismo del gen de la MTHFR C677T (RR = 3; IC95\% 1,13-7,93; P 0,002), sugiriendo la necesidad de considerar las variaciones genéticas en futuros estudios ${ }^{29}$. Cuando se analizan en conjunto los polimorfismos de la MTHFR C677T y C1298A, se observa un significativo aumento del riesgo de cáncer de mama ( $\mathrm{RR}=2,17$; IC95\% $0,97-4,84 ; \mathrm{P} 0,01)$ en mujeres homocigotas para ambas variantes (TT y AA) con consumos elevados de folatos. Sin embargo, en heterocigotos CT y AC el riesgo disminuye con consumos elevados, sugiriendo la existencia de una conexión entre ambos polimorfismos ${ }^{30}$.

Ericson et $\mathrm{al}^{31}$ analizaron el riesgo considerando los receptores estrógenicos alfa y beta (ER $\alpha$ y ER $\beta$ ) de la mama, mostrando una asociación entre niveles elevados de folatos plasmáticos (>17 nmol/L) y cáncer $\operatorname{ER} \beta(-)$ con o sin polimorfismos de la MTFFR C677T, aún cuando se observa una tendencia positiva entre los portadores del alelo T (RR = 5,78; IC95\% 1,96-17,07; P0,01). A diferencia de estos resultados, Lin et $\mathrm{al}^{28}$ describió una asociación con $\operatorname{ER} \alpha(+)$, aún cuando en otros estudios no se describe un mayor riesgo asociado a estos receptores ${ }^{32-34}$.

Un efecto protector del folato se describe en mujeres consumidores de alcohol ${ }^{25,27}$, especialmente en mujeres post-menopaúsicas $(>15$ gramos/día) ${ }^{25}$, efecto que se mantiene tras ajustar el análisis por la ingesta de diferentes vitaminas ${ }^{26}$.

\section{Estudios de cohorte}

No todos los estudios seleccionados muestran una asociación significativa entre altos niveles de folatos y un menor riesgo de desarrollar cáncer de mama ${ }^{35-42}$. Algunos sugieren una asociación inversa entre ingestas moderadas de folatos y cáncer de $\mathrm{mama}^{43,44}$. Ericson $\mathrm{U}$ et $\mathrm{al}^{43}$ muestran una reducción en la incidencia de cáncer de mama cercana al $40 \%$ en los quintiles más altos de consumo de folatos dietarios y totales $(\mathrm{HR}=0,56$; IC $95 \% 0,35-0,90$ y RR = 0,56; IC 95\% 0,34-0,91) concluyendo que en mujeres postmenopáusicas, un mayor consumo se relacionaría con un menor riesgo. Maruti et a ${ }^{45}$ encontraron que el efecto protector de los folatos, asociado al consumo de ácido fólico, aún cuando el período de seguimiento fue corto (5,5 años) que podría limitar la observación de efectos adversos. Un seguimiento de población superior a 20 años muestra que las mujeres con niveles de folatos en el glóbulo rojo medidos en el cuartil superior de la distribución versus el cuartil inferior tienen un menor riesgo para morbilidad, pero no para mortalidad por cáncer de mama ${ }^{44}$.

A diferencia de lo descrito anteriormente, un estudio multicéntrico que investigó la relación entre folatos totales, folatos dietarios, suplementos y cáncer de mama en mujeres postmenopáusicas mostró un significativo aumento de riesgo con mayores consumos de folatos, especialmente de folatos totales. Las mujeres que consumían suplementos de ácido fólico (> $400 \mathrm{mg} /$ día) mostraban un mayor riesgo (19\%) en relación a aquellas sin consumo. Las mujeres pertenecientes al quintil superior de consumo de folatos totales mostraban $32 \%$ de mayor riesgo. Al considerar el período 


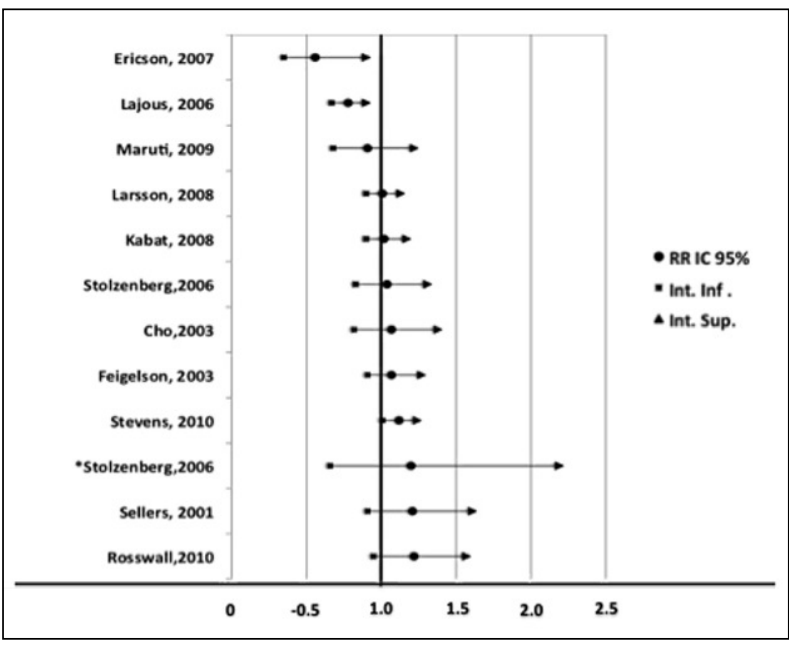

Figura 3. Ingesta de folatos dietarios y riesgo Ca mama. Estudios de cohorte. *Folatos dietarios sin uso de multivitaminas.

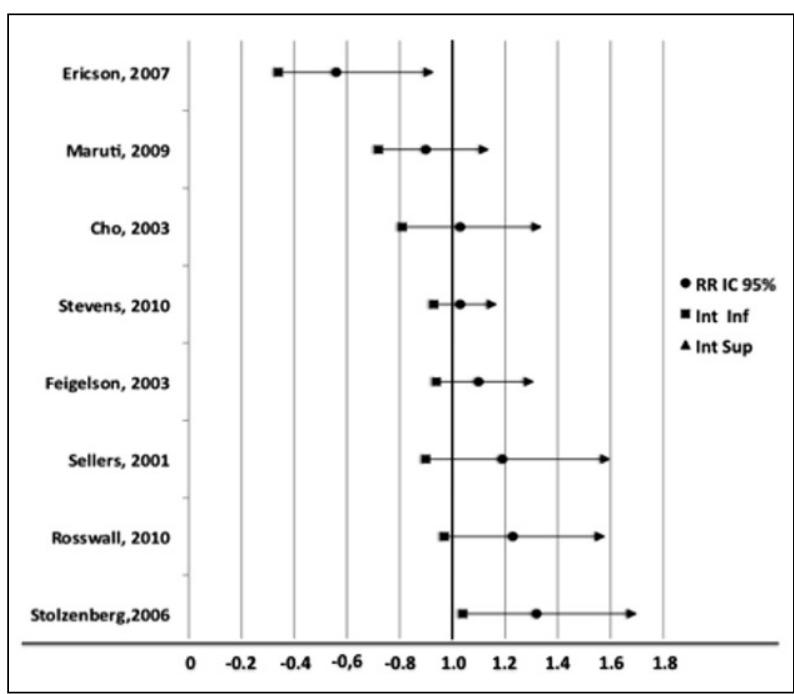

Figura 4. Ingesta de folatos totales y riesgo de cáncer de mama. Estudios de cohorte.

pre-fortificación de la harina, se observó que el mayor aporte de ácido fólico provenía de cereales fortificados y se asociaba también a un mayor riesgo (RR 1,69 IC 95\% $0,92-3,10)^{38}$ (Figura 3 y 4 ). Recientemente, un aumento de la ingesta de folatos dietarios también se ha asociado con un mayor riesgo de cáncer de mama en la menopausia ${ }^{32}$.

Cuando Larsson et $\mathrm{al}^{46}$ incorporaron en el análisis receptores hormonales, se observa que la ingesta de folatos en mujeres pre y post-menopaúsicas podría estar inversamente asociada en tumores con receptores positivo de estrógeno $(\mathrm{ER}+)$ y negativo de progesterona
(PR-) (RR = 0,78; IC 95\% 0,64-0,95 x c/100 $\mathrm{mg} /$ día de incremento de ácido fólico). Por otra parte, Maruti et al ${ }^{32}$ encuentran que el mayor beneficio se observaría con un mayor consumo de folato, incluyendo suplementos, cuando los receptores estrógenicos son negativos (ER-) ( $R R=0,38$; IC95\% 0,18-0,8; $\mathrm{P}$ 0,02). Una relación diferente se observa en un estudio reciente ${ }^{42}$, que muestra una asociación directa, aunque limitada sólo a folatos dietarios y tumores ER+ y PR+, pero no en tumores sólo ER-, semejante a lo descrito por Lin et $\mathrm{a}^{28}$.

El nivel de riesgo descrito para consumo de alcohol, folatos y cáncer de mama varía entre los diferentes estudios, mostrando que exisitiría un efecto protector con ingestas elevadas de folatos dietarios, especialmente en mujeres post-menopáusicas ${ }^{33,38,47,48}$. Algunos describen una protección débil ${ }^{39}$ cuando el consumo es elevado o bien no se demuestra un efecto protector ${ }^{37,40,46}$.

\section{Estudios clínicos aleatorios}

Un estudio clínico aleatorio doble ciego y controlado con placebo en 5.442 mujeres mayores de 42 años con enfermedad cardiovascular pre-existente que recibieron $2,5 \mathrm{mg}$ de ácido fólico, más $50 \mathrm{mg}$ de vitamina $\mathrm{B}_{6}$ y $1 \mathrm{mg}$ de vitamina $B_{12}$ concluye que no es posible determinar la existencia de beneficios o riesgos en relación a cáncer en general, cáncer de mama o muertes asociadas a cáncer ${ }^{49}$. Otro estudio, en 3.187 mujeres que recibieron 0,2 y $5 \mathrm{mg}$ de folatos/día durante 25 años muestra un aumento de la mortalidad general en el grupo que recibió folatos y un aumento no significativo en el riesgo de cáncer de mama $\left(\mathrm{HR}=2,02 ; \mathrm{IC} 95 \% 0,88-4,72 ; \mathrm{P} 0,1^{50}\right.$.

\section{Meta-análisis}

Un metanálisis que incluyó trece estudios caso-control muestra que una adecuada ingesta de folatos dietarios podría ser protectora contra el cáncer de mama $(\mathrm{OR}=0,91$; IC95\% 0,86-0,96) aunque la gran heterogeneidad de los estudios no permiten concluir que exista realmente una asociación ${ }^{51}$. Por otra parte, con los nueve estudios de cohorte examinados no se logra demostrar una asociación entre ingesta de folatos y riesgo de 
cáncer de mama $(\mathrm{RR}=0,99 ; \mathrm{IC} 95 \% 0,98-1,01)$. De los diecisiete estudios analizados que consideraron el genotipo MTHFR C677T tampoco se extrae ninguna interacción entre los heterocigotos y los homocigotos de este genotipo y el cáncer de mama, así como tampoco, con la ingesta de folatos ${ }^{51}$.

Otro metanálisis de ocho estudios de cohorte no observa la existencia de un mayor riesgo de cáncer en relación a folatos totales y folatos dietarios (RR 0,97; IC 95\% 0,87-1,07) y (RR 1,01 IC 95\% 0,97-1,05). En los 13 estudios caso-control considerados se muestra una asociación inversa para folato dietario $(\mathrm{OR}=0,8 ; \mathrm{IC} 95 \% 0,72-0,89)$ y para folato total $(\mathrm{OR}=0,93$; IC $95 \% 0,81-1,07)$. En los estudios de cohorte prospectivo y en los casocontrol que compararon altos niveles de folato sanguíneo versus bajos niveles tampoco se describe alguna asociación significativa, a excepción de las mujeres con consumo de alcohol elevado y un bajo consumo de folatos (RR 0,51; IC 95\% 0,41-0,63) . $^{52}$.

La relación entre algunos polimorfismos genéticos asociados al metabolismo del folato y el riesgo de cáncer de mama, en general, no muestran asociaciones significativas. Cuando se analizan subgrupos de población considerando los genotipos de la MTFHR 677CT y 1298 AC $^{53}$ sólo se observa un mayor riesgo de cáncer en mujeres premenopáusicas homocigotas para MTHFR 677TT (RR 1,49; IC 95\% 1,09-2,03). Un resultado similar muestran Macis el al ${ }^{54}$ en un metanálisis basado en dieciocho estudios caso control, con un aumento de riesgo del $40 \%$ en mujeres premenopáusicas y homocigotos (TT).

\section{Discusión}

Aunque el aumento de la ingesta de folatos pueda ser beneficioso para poblaciones con deficiencias, en mujeres con niveles adecuados de folatos podría no entregar beneficios adicionales, sino más bien constituir un riesgo ${ }^{38}$.

El cáncer de mama es una de las patologías más frecuentes en mujeres, determinando 5 a $7 \%$ de todas las muertes en países desarrollados. Estudios de experimentación en animales no muestran un beneficio adicional para tumores de mama cuando se aporta ácido fólico desde el inicio de la gestación, observándose sólo un efecto protector en ratas con dietas deficientes de folatos ${ }^{55}$. Sin embargo, estos resultados no son consistentes con los resultados de estudios epidemiológicos.
Un efecto protector asociado a ingestas elevadas de folatos dietarios se muestra en algunos estudios caso-control. Esta ingesta determinaría niveles séricos adecuados de 5 metil-THF y de la coenzima S-adenosil-metionina (SAM), asegurando así, una correcta metilación del ADN, un menor daño y una adecuada expresión de genes reparadores ${ }^{17}$. Sin embargo, en otros estudios se observa un mayor riesgo con niveles elevados, especialmente durante la premenopausia, etapa donde existe una mayor proliferación celular y una mayor expresión de receptores de folatos, que limitaría el tiempo para una adecuada reparación del $\mathrm{ADN}^{28}$. Sin embargo, los resultados de los estudios caso control deben ser analizados con precaución considerando que sus análisis retrospectivos pueden determinar imprecisiones en relación a la estimación de ingesta dietaria de folatos.

Los estudios de cohorte revisados describen riesgos que dependen del tipo de folatos, observándose en algunos que éste aumenta cuando la ingesta de folatos totales (folatos dietarios más suplementos de ácido fólico) es elevada. Esta mayor ingesta, especialmente como ácido fólico, podría contribuir a cambios epigenéticos en la regulación de la expresión de los genes ${ }^{18}$. Es importante destacar las diferencias existentes entre los diferentes estudios para definir ingesta elevada de folatos totales; por ejemplo, el estudio de Ericson et a ${ }^{30}$ utiliza valores de $456 \mathrm{mg} /$ día, mientras que Stolzenberg et $\mathrm{al}^{38}$ consideran valores $>853 \mathrm{mg} /$ día. Estas diferencias debieran ser consideradas y controladas en futuros estudios.

La existencia de polimorfismos de las enzimas participantes en el metabolismo del folato podrían explicar las diferencias observadas en el riesgo de cáncer de mama, especialmente en mujeres homocigotas (TT) de la MTHFR 677CT que presentan una menor actividad enzimática ${ }^{54}$. Ingestas elevadas de ácido fólico determinarían una acumulación de isómeros distintos a 5-MTHF, empujando esta vía metabólica hacia la síntesis de $\mathrm{ADN}$ en desmedro de la metilación. Cuando las ingestas de ácido fólico son elevadas, sólo un porcentaje podría reducirse a tetrahidrofolato activo, determinando que una parte se mantuviera inactiva, compitiendo con folatos naturales y alterando el metabolismo y el transporte de folatos al interior de las células ${ }^{27,28}$. Esto sugeriría que la relación entre folato y cáncer de mama dependería específicamente del polimorfismo analizado, 
sin tener un rol independiente en la etiología del cáncer de mama ${ }^{21}$.

Los meta-análisis publicados en relación folatos y riesgo de cáncer de mama tampoco son concluyentes para identificar riesgos o beneficios dada la heterogeneidad de los estudios ${ }^{50,51}$. Sólo se describe una asociación significativa para disminución de cáncer en mujeres con alto consumo de alcohol y bajo consumo de folatos. Sin embargo, cuando se estratifican por premenopausia y menopausia, la premenopausia aparece con un mayor riesgo para las mujeres homocigota $\mathrm{TT}^{53,54}$.

Al igual que lo descrito en otros tipos de cáncer, pareciera que la relación entre consumo de folatos y desarrollo de cáncer de mama no es lineal, siendo beneficioso un mayor aporte en poblaciones con consumos deficientes, pero determinante de mayores riesgos cuando la ingesta es elevada. Considerando la complejidad del metabolismo del folato, los futuros estudios destinados a identificar riesgos y beneficios debieran considerar las características genéticas de las poblaciones estudiadas, tipos y niveles de folatos consumidos, e identificar además interacciones con otras vitaminas, fotoquímicos y consumo de alcohol ${ }^{27}$.

También pareciera importante considerar en futuros estudios de folato y cáncer de mama, los diferentes tipos de receptores hormonales debido a la descripción de mayores riesgos en algunos $\operatorname{tipos}^{30-32}$. Destaca el mayor riesgo observado con ingestas bajas de folatos y tumores ER $\beta(-)$ cuya expresión es frecuente en células normales, pero que se observa disminuida en islas $\mathrm{CpG}$ metiladas de líneas celulares de tumores primarios y que se estimándo que podría actuar como supresor de tumores ${ }^{33,34}$.

Los estudios clínicos, aleatorios y controlados con placebos en relación a folato y cáncer de mama son limitados existiendo sólo una investigación que evalúa los efectos de la suplementación de ácido fólico en forma independiente y que describe un aumento del riesgo de cáncer asociada a ingestas elevadas, tendencia que podría corresponder al $\operatorname{azar}^{50}$ sugiriendo la necesidad de desarrollar nuevos estudios clínicos en relación a estas materias.

\section{Conclusión}

En resumen, en los artículos seleccionados para esta revisión existen grandes diferencias entre los tipos de folatos y también de ingesta dietaria y de folatos séricos considerados para el análisis que no permiten concluir la existencia de un menor riesgo de cáncer de mama asociado a un mayor nivel de consumo. Por otra parte, considerando la existencia de estudios que sugieren un mayor riesgo de cáncer de mama asociado a ingestas elevadas de folatos, especialmente como ácido fólico ${ }^{35,45}$ y en etapas posteriores a la fortificación obligatoria de alimentos, pareciera recomendable considerar la adopción de medidas precautorias que limiten la exposición de las mujeres a ingestas elevadas de ácido fólico.

\section{Referencias}

1. World Health Organization (WHO) Disponible en http://www.who.int/mediacentre/news/releases/2003/ pr27/en/ (Consultado el 27-11-2010).

2. National Cancer Institute (NCI). Cancers Trends Progress Report Disponible en: http://progressreport.cancer. gov/highlights.asp (Consultado el 27-9-2010).

3. Pollán M. Epidemiology of breast cancer in young women. Breast Cancer Res Treat 2010; 123 Suppl 1: 3-6.

4. $\mathrm{Xu} \mathrm{X,} \mathrm{Chen} \mathrm{J.} \mathrm{One-carbon} \mathrm{metabolism} \mathrm{and} \mathrm{breast} \mathrm{can-}$ cer: an epidemiological perspective. J Genet Genomics 2009; 36 (4): 203-14.

5. Jones PA, Baylin SB. The fundamental role of epigenetic events in cancer. Nat Rev Genet 2002; 3 (6): 415-28.

6. Castillo C, Tur Ja, Uauy R. [Flour fortification with folic acid in Chile. Unintended consequences]. Rev Med Chile 2010; 138 (7): 832-40.

7. Sánchez H, Albala C, Hertramps E, Verdugo R, Lavados $\mathrm{M}$, Castillo Jl, et al. [Prevalence of vitamin B-12 deficiency in older adults]. Rev Med Chile2010; 138 (1): 44-52.

8. Hirsch S, De La Maza P, Barrera G, Gattás V, Petermann $\mathrm{M}$, Bunout D. The Chilean flour folic acid fortification program reduces serum homocysteine levels and masks vitamin B-12 deficiency in elderly people. J Nutr 2002; 132 (2): 289-91.

9. CASPe (2011) Critical Appraisal Skills Programme Español (CASPe) [homepage]. Alicante, Spain: CASPe; (Consultado el 1 de marzo de 2011). Disponible en http://www.redcaspe.org

10. Chen J, Gammon MD, Chan W, Palomeque C, Wetmur JG, Kabat GC, et al. One-carbon metabolism, MTHFR polymorphisms, and risk of breast cancer. Cancer Res 2005; 65 (4): 1606-14.

11. Ma E, Iwasaki M, Kobayashi M, Kasuga Y, Yokoyama S, Onuma H, et al. Dietary intake of folate, vitamin B2, vitamin B6, vitamin B12, genetic polymorphism of related 
enzymes, and risk of breast cancer: a case-control study in Japan. Nutr Cancer 2009; 61(4): 447-56.

12. Ma E, Iwasaki M, Junko I, Hamada GS, Nishimoto IN, et al. Dietary intake of folate, vitamin B6, and vitamin B12, genetic polymorphism of related enzymes, and risk of breast cancer: a case-control study in Brazilian women. BMC Cancer 2009; 9: 122.

13. Sharp L, Little J, Schofield AC, Pavlidou E, Cotton SC, Miedzybrodzka Z, et al. Folate and breast cancer: the role of polymorphisms in methylenetetrahydrofolate reductase (MTHFR). Cancer Lett 2002; 181 (1): 65-71

14. Chou YC, Wu MH, Yu JC, Lee MS, Yang T, Shih HL, et al. Genetic polymorphisms of the methylenetetrahydrofolate reductase gene, plasma folate levels and breast cancer susceptibility: a case-control study in Taiwan. Carcinogenesis 2006; 27 (11): 2295-300.

15. Suzuki T, Matsuo K, Hirose K, Hiraki A, Kawase T, Watanabe $\mathrm{M}$, et al. One-carbon metabolism-related gene polymorphisms and risk of breast cancer. Carcinogenesis 2008 ; 29 (2): 356-62.

16. Levi F, Pasche C, Lucchini F, La Vecchia C. Dietary intake of selected micronutrients and breast-cancer risk. Int J Cancer 2001; 91 (2): 260-3.

17. Shrubsole MJ, Jin F, Dai Q, Shu XO, Potter JD, Hebert JR, et al. Dietary folate intake and breast cancer risk: results from the Shanghai Breast Cancer Study. Cancer Res 2001; 61 (19): 7136-41.

18. Lajous M, Lazcano-Ponce E, Hernández-Ávila M, Willett $\mathrm{W}$, Romieu I. Folate, vitamin $\mathrm{B}(6)$, and vitamin $\mathrm{B}(12)$ intake and the risk of breast cancer among Mexican women. Cancer Epidemiol Biomarkers Prev 2006; 15 (3): 443-8.

19. Beilby J, Ingram D, Hähnel R, Rossi E. Reduced breast cancer risk with increasing serum folate in a case-control study of the C677T genotype of the methylenetetrahydrofolate reductase gene. Eur J Cancer 2004; 40 (8): 1250-4.

20. Shrubsole MJ, Gao YT, Cai Q, Shu XO, Dai Q, Hébert JR, et al. MTHFR polymorphisms, dietary folate intake, and breast cancer risk: results from the Shanghai Breast Cancer Study. Cancer Epidemiol Biomarkers Prev 2004; 13 (2): 190-6.

21. Shrubsole MJ, Gao YT, Cai Q, Shu XO, Dai Q, Jin F, et al. MTR and MTRR polymorphisms, dietary intake, and breast cancer risk. Cancer Epidemiol Biomarkers Prev 2006; 15 (3): 586-8.

22. Zhu K, Davidson NE, Hunter S, Yang X, Payne-Wilks $\mathrm{K}$, Roland CL, et al. Methyl-group dietary intake and risk of breast cancer among African-American women: a case-control study by methylation status of the estrogen receptor alpha genes. Cancer Causes Control
2003;14(9):827-36.

23. Negri E, La Vecchia C, Franceschi S. Re: dietary folate consumption and breast cancer risk. J Natl Cancer Inst 2000; 92 (15): 1270-1.

24. Adzersen KH, Jess P, Freivogel KW, Gerhard I, Bastert G. Raw and cooked vegetables, fruits, selected micronutrients, and breast cancer risk: a case-control study in Germany. Nutr Cancer 2003;46(2):131-7.

25. Rohan TE, Jain MG, Howe GR, Miller AB. Dietary folate consumption and breast cancer risk. J Natl Cancer Inst 2000; 92 (3): 266-9.

26. Tjønneland A, Christensen J, Olsen A, Stripp C, Nissen $\mathrm{SB}$, Overvad K, et al. Folate intake, alcohol and risk of breast cancer among postmenopausal women in Denmark. Eur J Clin Nutr 2006; 60 (2): 280-6.

27. Zhang SM, Willett WC, Selhub J, Hunter DJ, Giovannucci EL, Holmes MD, et al. Plasma folate, vitamin B6, vitamin B12, homocysteine, and risk of breast cancer. J Natl Cancer Inst 2003; 95 (5): 373-80.

28. Lin J, Lee IM, Cook NR, Selhub J, Manson JE, JE, et al. Plasma folate, vitamin B-6, vitamin B-12, and risk of breast cancer in women. Am J Clin Nutr 2008;87(3):734-43.

29. Ericson UC, Ivarsson MI, Sonestedt E, Gullberg B, Carlson J, Olsson $\mathrm{H}$, et al. Increased breast cancer risk at high plasma folate concentrations among women with the MTHFR 677T allele. Am J Clin Nutr 2009; 90 (5): 1380-9.

30. Ericson U, Sonestedt E, Ivarsson MI, Gullberg B, Carlson J, Olsson $\mathrm{H}$, et al. Folate intake, methylenetetrahydrofolate reductase polymorphisms, and breast cancer risk in women from the Malmö Diet and Cancer cohort. Cancer Epidemiol Biomarkers Prev 2009; 18 (4): 1101-10.

31. Ericson U, Borgquist S, Ivarsson MI, Sonestedt E, Gullberg B, Carlson J, et al. Plasma folate concentrations are positively associated with risk of estrogen receptor beta negative breast cancer in a Swedish nested case control study. J Nutr 2010; 140 (9): 1661-8.

32. Maruti SS, Ulrich CM, White E. Folate and one-carbon metabolism nutrients from supplements and diet in relation to breast cancer risk. Am J Clin Nutr 2009; 89 (2): 624-33.

33. Sellers TA, Alberts SR, Vierkant RA, Grabrick DM, Cerhan JR, Vachon CM, et al. High-folate diets and breast cancer survival in a prospective cohort study. Nutr Cancer 2002; 44 (2): 139-44.

34. Zhang SM, Hankinson SE, Hunter DJ, Giovannucci EL, Colditz GA, Willett WC. Folate intake and risk of breast cancer characterized by hormone receptor status. Cancer Epidemiol Biomarkers Prev 2005; 14 (8): 2004-8.

35. Sellers TA, Kushi LH, Cerhan JR, Vierkant RA, Gapstur 
SM, Vachon CM, et al. Dietary folate intake, alcohol, and risk of breast cancer in a prospective study of postmenopausal women. Epidemiology 2001; 12 (4): 420-8.

36. Cho E, Spiegelman D, Hunter DJ, Chen WY, Zhang SM, Colditz GA, et al. Premenopausal intakes of vitamins $\mathrm{A}, \mathrm{C}$, and $\mathrm{E}$, folate, and carotenoids, and risk of breast cancer. Cancer Epidemiol Biomarkers Prev 2003; 12 (8): 713-20.

37. Feigelson HS, Jonas CR, Robertson AS, Mccullough ML, Thun MJ, Calle EE. Alcohol, folate, methionine, and risk of incident breast cancer in the American Cancer Society Cancer Prevention Study II Nutrition Cohort. Cancer Epidemiol Biomarkers Prev 2003; 12 (2): 161-4.

38. Stolzenberg-Solomon RZ, Chang SC, Leitzmann MF, Johnson KA, Johnson C, Buys SS, et al. Folate intake, alcohol use, and postmenopausal breast cancer risk in the Prostate, Lung, Colorectal, and Ovarian Cancer Screening Trial. Am J Clin Nutr 2006; 83 (4): 895-904

39. Kabat GC, Miller AB, Jain M, Rohan TE. Dietary intake of selected $\mathrm{B}$ vitamins in relation to risk of major cancers in women. Br J Cancer 2008; 99 (5): 816-21.

40. Duffy CM, Assaf A, Cyr M, Burkholder G, Coccio E, Rohan $\mathrm{T}$, et al. Alcohol and folate intake and breast cancer risk in the WHI Observational Study. Breast Cancer Res Treat 2009; 116 (3): 551-62.

41. Larsson SC, Bergkvist L, Wolk A. Folate intake and risk of breast cancer by estrogen and progesterone receptor status in a Swedish cohort. Cancer Epidemiol Biomarkers Prev2008; 17 (12): 3444-9.

42. Roswall N, Olsen A, Christensen J, Dragsted LO, Overvad K, Tjønneland A. Micronutrient intake and breast cancer characteristics among postmenopausal women. Eur J Cancer Prev 2010; 19 (5): 360-5.

43. Ericson U, Sonestedt E, Gullberg B, Olsson H, Wirfält E. High folate intake is associated with lower breast cancer incidence in postmenopausal women in the Malmö Diet and Cancer cohort. Am J Clin Nutr 2007; 86 (2): 434-43.

44. Rossi E, Hung J, Beilby JP, Knuiman MW, Divitini ML, Bartholomew H. Folate levels and cancer morbidity and mortality: prospective cohort study from Busselton, Western Australia. Ann Epidemiol 2006; 16 (3): 206-12.

45. Stevens VL, Mccullough ML, Sun J, Gapstur SM. Folate and other one-carbon metabolism-related nutrients and risk of postmenopausal breast cancer in the Cancer Prevention Study II Nutrition Cohort. Am J Clin Nutr 2010; 91 (6): 1708-15.

46. Larsson SC, Bergkvist L, Wolk A. Folate intake and risk of breast cancer by estrogen and progesterone receptor status in a Swedish cohort. Cancer Epidemiol Biomarkers Prev 2008; 17 (12): 3444-9.

47. Baglietto L, English DR, Gertig DM, Hopper JL, Giles GG. Does dietary folate intake modify effect of alcohol consumption on breast cancer risk? Prospective cohort study. BMJ 2005; 331 (7520): 807.

48. Lajous M, Romieu I, Sabia S, Boutron-Ruault MC, Clavel-Chapelon F. Folate, vitamin B12 and postmenopausal breast cancer in a prospective study of French women. Cancer Causes Control 2006; 17 (9): 1209-13.

49. Zhang SM, Cook NR, Albert CM, Gaziano JM, Buring JE, Manson JE. Effect of combined folic acid, vitamin B6, and vitamin $\mathrm{B} 12$ on cancer risk in women: a randomized trial. JAMA 2008; 300 (17): 2012-21.

50. Charles D, Ness AR, Campbell D, Davey Smith G, Hall $\mathrm{MH}$. Taking folate in pregnancy and risk of maternal breast cancer. BMJ 2004; 329 (7479): 1375-6.

51. Lewis SJ, Harbord RM, Harris R, Smith GD. Metaanalyses of observational and genetic association studies of folate intakes or levels and breast cancer risk. J Natl Cancer Inst 2006; 98 (22): 1607-22.

52. Larsson SC, Giovannucci E, Wolk A. Folate and risk of breast cancer: a meta-analysis. J Natl Cancer Inst 2007; 99 (1): 64-76 102.

53. Zintzaras E. Methylenetetrahydrofolate reductase gene and susceptibility to breast cancer: a meta-analysis. Clin Genet 2006; 69 (4): 327-36.

54. Macis D, Maisonneuve P, Johansson H, Bonanni B, Botteri E, Iodice S, et al. Methylenetetrahydrofolate reductase (MTHFR) and breast cancer risk: a nestedcase-control study and a pooled meta-analysis. Breast Cancer Res Treat 2008; 107 (3): 459-60. 2007110.

55. Kotsopoulos J, Medline A, Renlund R, Sohn KJ, Martin R, Hwang SW, et al. Effects of dietary folate on the development and progression of mammary tumors in rats. Carcinogenesis 2005; 26 (9): 1603-12. 